\title{
Reproductive justice in the time of COVID-19: a systematic review of the indirect impacts of COVID-19 on sexual and reproductive health
}

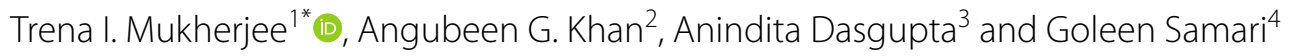

\begin{abstract}
Objective: Despite gendered dimensions of COVID-19 becoming increasingly apparent, the impact of COVID-19 and other respiratory epidemics on women and girls' sexual and reproductive health (SRH) have yet to be synthesized. This review uses a reproductive justice framework to systematically review empirical evidence of the indirect impacts of respiratory epidemics on SRH.
\end{abstract}

Methods: We searched MEDLINE and CINAHL for original, peer-reviewed articles related to respiratory epidemics and women and girls'SRH through May 31, 2021. Studies focusing on various SRH outcomes were included, however those exclusively examining pregnancy, perinatal-related outcomes, and gender-based violence were excluded due to previously published systematic reviews on these topics. The review consisted of title and abstract screening, fulltext screening, and data abstraction.

Results: Twenty-four studies met all eligibility criteria. These studies emphasized that COVID-19 resulted in service disruptions that effected access to abortion, contraceptives, HIV/STI testing, and changes in sexual behaviors, menstruation, and pregnancy intentions.

Conclusions: These findings highlight the need to enact policies that ensure equitable, timely access to quality SRH services for women and girls, despite quarantine and distancing policies. Research gaps include understanding how COVID-19 disruptions in SRH service provision, access and/or utilization have impacted underserved populations and those with intersectional identities, who faced SRH inequities notwithstanding an epidemic. More robust research is also needed to understand the indirect impact of COVID-19 and epidemic control measures on a wider range of SRH outcomes (e.g., menstrual disorders, fertility services, gynecologic oncology) in the long-term.

Keywords: COVID-19, Reproductive health, Sexual health, Gender, Health inequity, Abortion, Contraceptives

\section{Plain English summary}

The impact of respiratory epidemics, like COVID-19 on women and girls'sexual and reproductive health (SRH) is not yet known. This review applies a reproductive justice framework, to systematically review the impact of respiratory

*Correspondence: tm2925@cumc.columbia.edu

1 Department of Epidemiology, Mailman School of Public Health, Columbia University, New York, NY, USA

Full list of author information is available at the end of the article

(c) The Author(s) 2021. Open Access This article is licensed under a Creative Commons Attribution 4.0 International License, which permits use, sharing, adaptation, distribution and reproduction in any medium or format, as long as you give appropriate credit to the original author(s) and the source, provide a link to the Creative Commons licence, and indicate if changes were made. The images or other third party material in this article are included in the article's Creative Commons licence, unless indicated otherwise in a credit line to the material. If material is not included in the article's Creative Commons licence and your intended use is not permitted by statutory regulation or exceeds the permitted use, you will need to obtain permission directly from the copyright holder. To view a copy of this licence, visit http://creativecommons.org/licenses/by/4.0/. The Creative Commons Public Domain Dedication waiver (http://creativeco mmons.org/publicdomain/zero/1.0/) applies to the data made available in this article, unless otherwise stated in a credit line to the data. 
epidemics on SRH, in order to examine the impact of COVID-19 on equitable, sustained access to quality SRH services for all populations. This framework highlights the right to reproductive autonomy, including the right to have an abortion, conceive, bear and raise children; and is inclusive of the intersectionality of race, class and gender. This review includes original, peer-reviewed research related to COVID-19 and women and girls'SRH through May 31, 2021, and consisted of title and abstract screening, full-text screening, and data abstraction. Overall, twenty-four studies met eligibility criteria. Results emphasize that the COVID-19 pandemic resulted in service disruptions that effected access to abortion, contraceptives, HIV/STI testing, and changes in changes in sexual behaviors, menstruation, and pregnancy intentions. These findings highlight the urgent need to enact policies that ensure equitable, timely access to quality SRH services for women and girls, despite pandemic response policies. This review also highlights opportunities to better understand how COVID-19 related disruptions in SRH service provision, access and/or utilization have impacted underserved populations and those with intersectional identities, who faced SRH inequities prior to the COVID-19 pandemic. More research is needed to understand the indirect impact of COVID-19 and epidemic control measures on a wider range of SRH outcomes (e.g., menstrual disorders, fertility services, gynecologic oncology) in the long-term.

\section{Background}

As the COVID-19 pandemic continues to take lives worldwide, an understanding of the short- and long-term consequences of the pandemic on women's and girls' sexual and reproductive health (SRH) is critical $[1,2]$. Global responses, lockdowns, and travel restrictions converge with pervasive, existing health inequities and injustices to disproportionately impact the health, wellbeing, and economic stability of women and girls [3]. The indirect consequences of COVID-19 control may be overlooked in the immediate need to mitigate transmission, and SRH-related morbidity and mortality will not become apparent for years to come. Several commentaries have discussed the disruptions to SRH care provision that providers and family planning clinics experienced $[1,2,4$, 5]; including interruptions to the supply and provision of contraception, abortion and post-abortion care, a decline in the number of patients served due to inaccessibility, and reduced client engagement as lockdowns and travel restrictions went into effect [6].

In light of the efforts to exclude SRH from essential health services during COVID-19 [1-3, 7], an understanding of the impact of the COVID-19 pandemic on SRH is critical for informing future actions and policies that prevent adverse SRH outcomes and comorbidities. Evidence from the SARS, MERS and Ebola pandemics envisage that the populations for whom human rights are least protected and most violated (e.g. women/girls, youth, poor people, immigrants, racial/ethnic minorities) will experience severe, unique difficulties and differentially die from COVID-19 $[8,9]$. Even prior to the COVID-19 pandemic, African American/Black, Latinx, immigrant, and women and girls with lower socioeconomic status experienced greater SRH disparities [1013]. The restrictions on movement disproportionately affect marginalized populations, and simulation studies estimate that COVID-19 related disruptions in essential SRH care will result in declines in short- and long-acting reversible contraceptive use, and increases in unintended pregnancies and unsafe abortions [14]. Conservative estimates of the impact of service disruptions at Marie Stopes International-affiliated health facilities across 37 countries suggest that the COVID-19 pandemic could result in 1.3 million unintended pregnancies, 1.2 million unsafe abortions, and 5000 pregnancy-related deaths [6]. Therefore, it is crucial to apply a reproductive justice framework to ensure equitable, sustained access to quality SRH services for all populations throughout the duration of the COVID-19 pandemic. This framework highlights the right to reproductive autonomy, including the right to have an abortion, and to conceive, bear and raise children; and is inclusive of the intersectionality of race, class and gender $[15,16]$.

Despite hypothesized impacts, empirical evidence of the indirect impacts of the COVID-19 pandemic on women and girls SRH have yet to be synthesized. We apply a reproductive justice framework to systematically review empirical evidence on the indirect impacts of the COVID19 pandemic on women and girls' SRH, in order to identify the observed effects of COVID-19 and the pandemic response on SRH; and to highlight SRH disparities for marginalized women and girls who are all too often overlooked and underserved.

\section{Main text}

\section{Methods}

A protocol with search terms was developed in consultation with and approved by a trained systematic review specialist at Columbia University. Respiratory illness related search terms included "pandemic, epidemic, outbreak, influenza, COVID-19, coronavirus, 1918 Flu, Middle Eastern Respiratory Syndrome, MERS, Severe Acute Respiratory Syndrome, SARS, Swine Flu, and H1N1." 
Outlined by the reproductive justice framework $[15,16]$, with a focus on reproductive autonomy, including the right to have an abortion, and to conceive, bear and raise children, SRH search terms included "preventative and curative care related to pregnancy, fertility, contraception, sexually transmitted infection (STI), reproductive cancers and other reproductive morbidities, genderbased, gender inequities, women's health, sexual health, reproductive health, obstetric, gynecol*, pregnancy, fertility, contracepti*, abortion, family planning, STI/STD, sexual violence, maternal health, reproductive coercion, maternal mortality, reproductive justice, menstrual hygiene, and reproductive tract infection."

Peer-reviewed studies published until May 31, 2021 were included from journals across MEDLINE via PubMed and CINAHL (PsychINFO, Gender Studies Database, Violence \& Abuse, Women's Studies International). Inclusion criteria included respiratory illness epidemic and an outcome explicit to women and girls' SRH. Populations could have been diagnosed with, exposed to, or impacted by public health responses (i.e., service disruptions, lockdowns, etc.) to respiratory epidemics or pandemics. Studies also had to have abstracts, full-texts and be published in a peer-reviewed journal. Articles without English translation, opinion pieces, commentaries, guidelines and simulation/modelling studies were excluded. Those addressing non-respiratory epidemics (i.e., obesity, opioid, HIV, etc.) and those that failed to examine SRH outcomes beyond vaccine interest and/or the psychological and emotional impact of the pandemic among pregnant women were excluded. Although pregnancy and birth-related outcomes, gender-based violence, and maternal and child health fall within the realm of reproductive justice, studies exclusively examining these outcomes were excluded, as systematic reviews including these topics have been recently published elsewhere [17-28]. Studies could have been published in any country including low, middle, and high-income settings, and there was no restriction on study publication dates.

The review consisted of screening: (1) titles, (2) abstracts, (3) full-texts, (4) data abstraction, and (5) critical appraisal of study bias. Each phase was completed independently by study authors. Title, abstract, and full text screening of eligible articles were completed by TM, AK, AD, and GS. Study data (author, study type, epidemic, SRH outcome and major findings) were abstracted by AK and TM. Data-screening procedures were applied according to the eligibility criteria. At the data abstraction stage, reviewers used data collection forms to capture the primary epidemic and primary outcome measure(s), in addition to supplementary information on study design, sampling/data sources, analytical methods, and effect estimates. Studies that met all eligibility criteria were assessed for methodological quality and risk of bias using the Quality Assessment Tool for Studies with Diverse Designs (QATSDD) as it enables review of studies with similar research questions, but different study designs. The QATSSD has shown good reliability and validity for quantitative and qualitative study designs $[29,30]$. The QATSDD consists of 16-items (4-items are for quantitative or qualitative studies only) that are rated on a 4-point Likert Scale. Total scores range from 0 to 42 , with higher scores indicating higher quality research. Scores were converted into a percentage, and those scoring $>60 \%$ were rated as high-quality studies, whereas those scoring $\leq 60 \%$ were rated as lower quality studies [31]. TM and AK independently reviewed and rated each study for risk of bias. Any disagreements were discussed until an agreement was reached.

\section{Results}

The search returned 2913 unique articles for title and abstract review, of which 88 met eligibility criteria and were included for full text review (Fig. 1). Twentyfour articles met all eligibility criteria after full review (Tables 1, 2). Most $(n=22,92 \%)$ were quantitative, with over half using cross-sectional $(\mathrm{n}=13,55 \%)$ study designs. The majority of studies were published in the global North $(n=16,67 \%)$, and all examined the impact of COVID-19. No studies examined the impact of the 1918 Flu, H1N1 and SARS or Middle Eastern Respiratory Syndrome (MERS) on non-pregnancy related SRH outcomes. One-third of studies examined SRH outcomes related to abortion $(\mathrm{n}=8,33 \%)$, one-quarter examined changes in service provision $(n=6,25 \%)$, while others examined contraceptive access or utilization $(n=5$, $21 \%)$, sexual behavior $(\mathrm{n}=4,17 \%)$, pregnancy intentions $(\mathrm{n}=3,13 \%)$, and menstrual cycle changes $(\mathrm{n}=2,8 \%)$. The majority $(\mathrm{n}=14,58 \%)$ of studies were rated as having low methodological quality based on the total QATSDD score, with total scores ranging from 31 to $88 \%$.

\section{Abortion}

The majority of abortion-related studies report results exclusively from the US $(n=6,75 \%)$. Across the US, the overall number of abortions decreased, however, demand for self-managed medication abortions increased during pandemic-related lockdowns and in the period immediately following lockdown. This was especially pronounced in states with greater stay-athome orders, or in states with more restrictive abortion policies [32]. The need for in-person visits for medication abortion decreased from two visits among most providers (71\%) to no in-person visits among $50 \%$ of abortion providers surveyed across the US [33]. Telemedicine, with in-person medication pick up or mail 


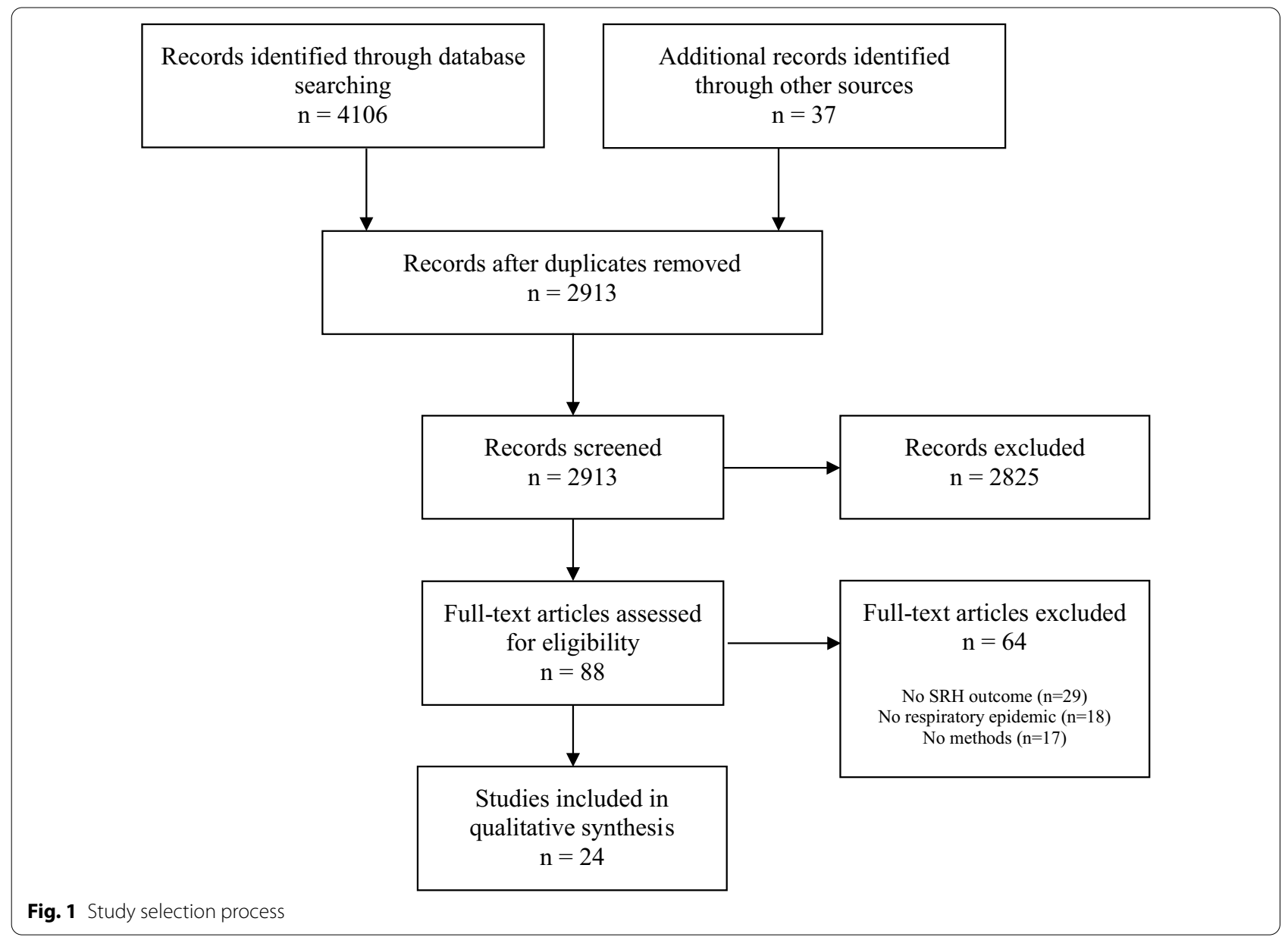

order was found to be acceptable during the pandemic in Hawaii, and was found to have high rates of success, follow-up retention and very little complications [34]. In a separate study of 103 abortion clinics across the US, several providers reported having to postpone, cancel or temporarily close their clinics due to staff being sick with probable COVID-19, COVID-19 related travel restrictions, or caregiving responsibilities, especially in the Southern states [35]. In Texas, an executive order postponing all unnecessary medical procedures (including abortion) prohibited most abortion procedures. Consequently, the total number of abortions fell by $38 \%$, the number of out of state abortions increased by over $500 \%$, and medication abortions increased by $41 \%$ [36]. Similarly, the number of medication abortions peaked at the height of COVID-19 in other states with restrictive abortion policies (Ohio, Kentucky \& West Virginia) [37].

Similarly, the number of surgical and medication abortions decreased globally, due to fear of COVID19, lack of transportation and access to pharmacies. Moreover, countries with restrictive abortion policies reported fewer women accessing abortion services, and fewer policy changes deeming SRH as essential to increase access to abortion or contraceptives during the pandemic [38]. A study in Nepal reported decreased demand for abortions during COVID-19 lockdown, which later increased once lockdowns were eased. Women who did receive abortions came in at a later gestational period and reported living closer to a health facility [39].

\section{Contraceptive access \& utilization}

All studies examining the impact of the COVID-19 pandemic and associated lockdowns on contraceptive access and utilization reported substantial decreases. Nearly all SRH-related clinicians, researchers and practitioners surveyed from 29 different countries reported that access to contraceptives and other SRH-related services decreased, primarily due to the prioritization of the pandemic response over SRH. A few respondents from high-income countries reported that the pandemic provided an opportunity to expand access to medication abortion, through telehealth services 
Table 1 Summary of studies included $(\mathrm{N}=24)$

\begin{tabular}{|c|c|}
\hline Characteristic & n (\%) \\
\hline \multicolumn{2}{|l|}{ Study design } \\
\hline Quantitative & $22(91.7)$ \\
\hline Cross-sectional & $13(54.2)$ \\
\hline Longitudinal & $3(12.5)$ \\
\hline Quasi-experimental & $2(8.3)$ \\
\hline Retrospective & $4(16.7)$ \\
\hline Mixed methods & $2(8.3)$ \\
\hline \multicolumn{2}{|l|}{ Sample size } \\
\hline None given & $1(4.2)$ \\
\hline $1-100$ & $7(29.2)$ \\
\hline $101-1000$ & $10(41.6)$ \\
\hline$>1000$ & $6(25.0)$ \\
\hline \multicolumn{2}{|l|}{ Region (as defined by WHO) } \\
\hline Africa & $1(4.2)$ \\
\hline Americas & $11(45.8)$ \\
\hline South-East Asia & $1(4.2)$ \\
\hline Europe & $5(20.8)$ \\
\hline Eastern Mediterranean & $0(0.0)$ \\
\hline Western Pacific & $2(8.3)$ \\
\hline Global & $2(8.3)$ \\
\hline \multicolumn{2}{|l|}{ Respiratory epidemic } \\
\hline COVID-19 & $24(100)$ \\
\hline \multicolumn{2}{|l|}{ Primary SRH outcome ${ }^{\mathrm{a}}$} \\
\hline Abortion & $8(33.3)$ \\
\hline Contraceptive access/utilization & $5(20.8)$ \\
\hline Menstruation & $2(8.3)$ \\
\hline Service provision & $6(25.0)$ \\
\hline Sexual behavior & $4(16.7)$ \\
\hline Pregnancy intentions & $3(12.5)$ \\
\hline \multicolumn{2}{|l|}{ QATSDD } \\
\hline High & $10(41.7)$ \\
\hline Low & $14(58.3)$ \\
\hline
\end{tabular}

SRH sexual and reproductive health; COVID-19 coronavirus disease 2019, WHOWorld Health Organization; QATSDD Quality Assessment Tool for Studies with Diverse Designs

${ }^{a}$ May not equate to $100 \%$ due to multiple outcomes

[38]. In the US, most family planning providers (91\%) reported providing telemedicine services for contraceptive counseling and prescriptions throughout the pandemic, with over half (53\%) making referrals to a minority of patients for in-person services for LARC insertion/removal, Depo-Provera injections or other contraceptive-related issues [40]. An ecological study using insurance data from a national database in France found that prescriptions of contraceptives and of ovulation indicators initially increased by $47 \%$ and $16 \%$ in the first 2-weeks of lockdown, but then substantially decreased. The decrease was sustained in the 4-weeks post-lockdown [41]. Pandemic related lockdowns contributed to a $20 \%$ decrease in contraceptive uptake in rural Mozambique. Once the lockdown was eased, however, contraceptive referrals by community health workers increased by $18 \%$. Moreover, uptake increased by $47 \%$ among women who were not currently using contraceptives, and by $80 \%$ by women who did not have phone access, and were likely of lower socioeconomic status [42].

In Northern Italy, where the majority of respondents reported using short-acting reversible contraceptives (SARC), half of all women who were not married/cohabiting discontinued their SARC during COVID-19 [43]. Of these, one-third reported an unintended pregnancy and sought an abortion. In Turkey, respondents reported a $14 \%$ decrease in contraceptive use, despite decreased desire for pregnancy, and increased sexual intercourse and menstrual disorders during COVID19 [44]. In China, 9\% of young women reported experiencing a shortage in contraceptives [45]. Similarly, few women (9\%) reported difficulties accessing contraceptives in Australia, however, nearly a quarter (22\%) reported unmet SRH-related needs, which include needing to access general practice, SRH specialist providers, pharmacies, hospitals, or counseling services [46]. In Nepal, 48\% of women seeking safe abortion services reported an increased need for contraception, with $23 \%$ not using contraceptives due to inaccessibility because of lockdowns [39]. The type of contraceptive was not noted in these studies, however.

Only one study reported $\mathrm{racial} / \mathrm{ethnic}$ disparities in contraceptive access. In the US, Black/African American, Latinx and Multi-racial respondents reported experiencing greater housing, transportation and food insecurity, when compared to White respondents. Poverty related factors of housing, transportation and food insecurity were found to be associated with an $86 \%$ greater difficulty in accessing contraceptives [47].

\section{Menstruation}

Only one study explicitly examined menstrual cycle changes [48], with almost half of all respondents reporting missed periods, with decreases and higher variability in cycle length. Yuksel et al. similarly report a $16 \%$ increase in menstrual disorders among survey respondents in Turkey [44]. The reason for menstrual cycle changes was not reported in either study, and it is not clear whether these changes were due to pandemic related lockdowns or COVID-19 infection. 


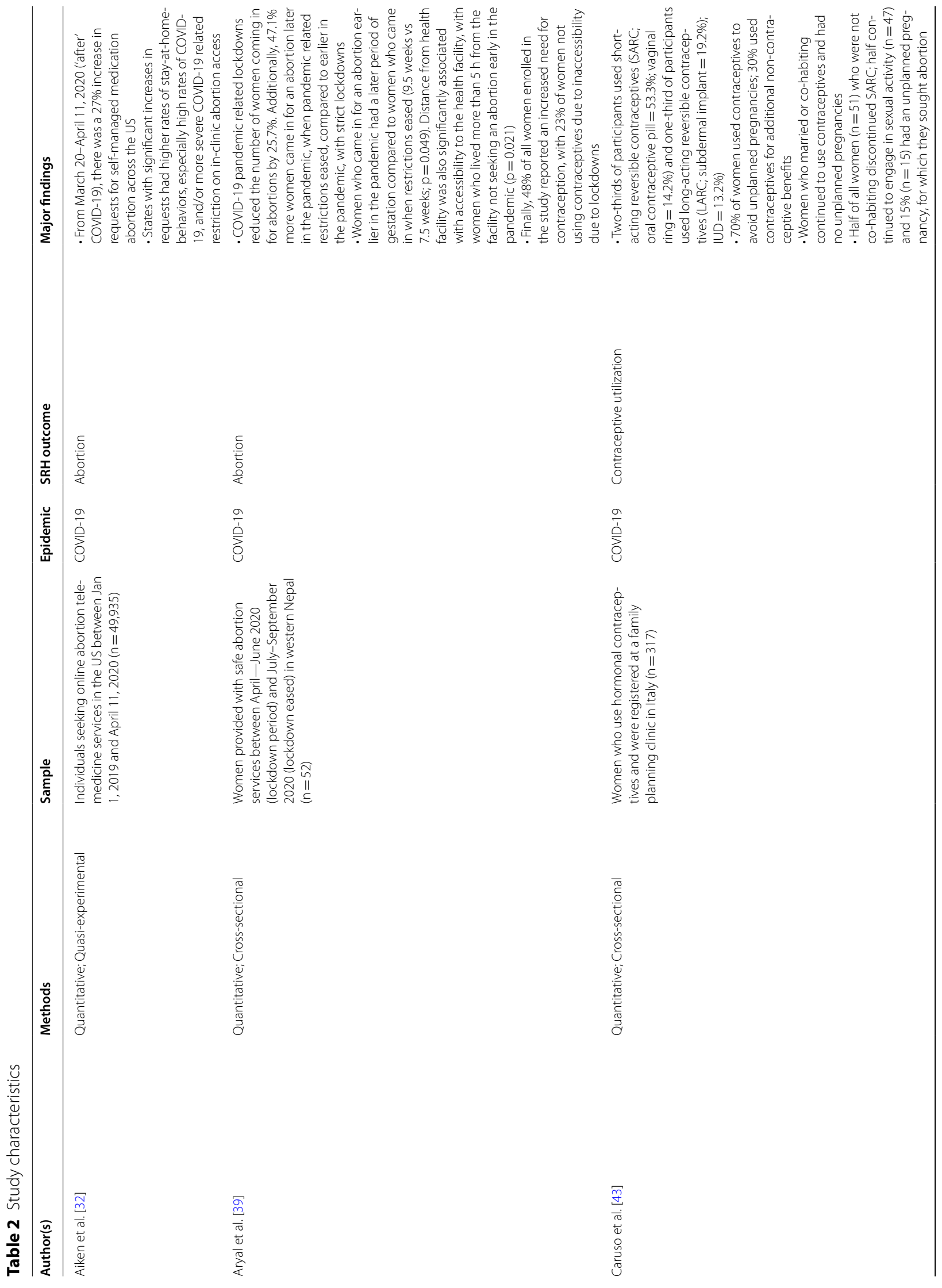




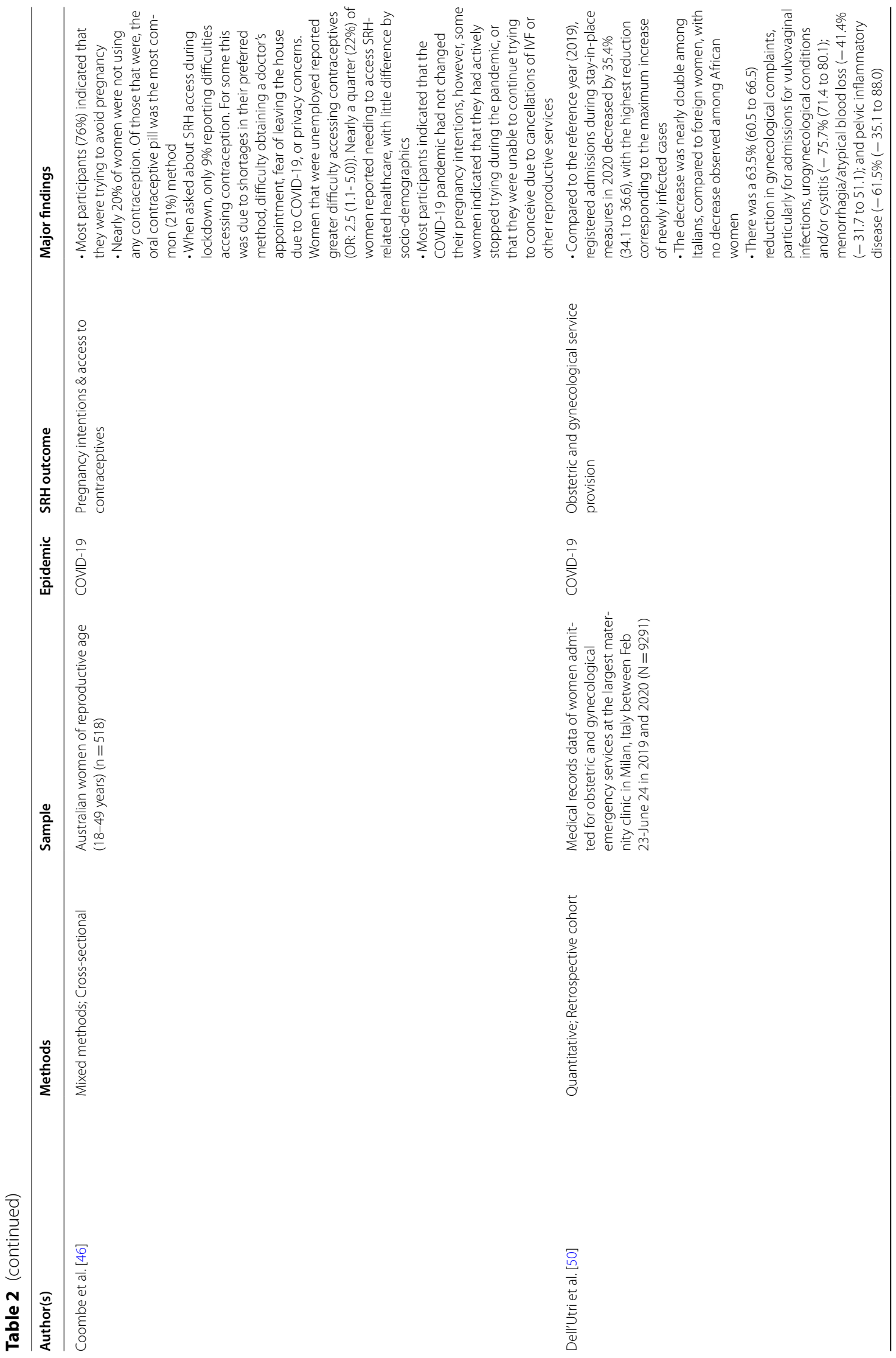




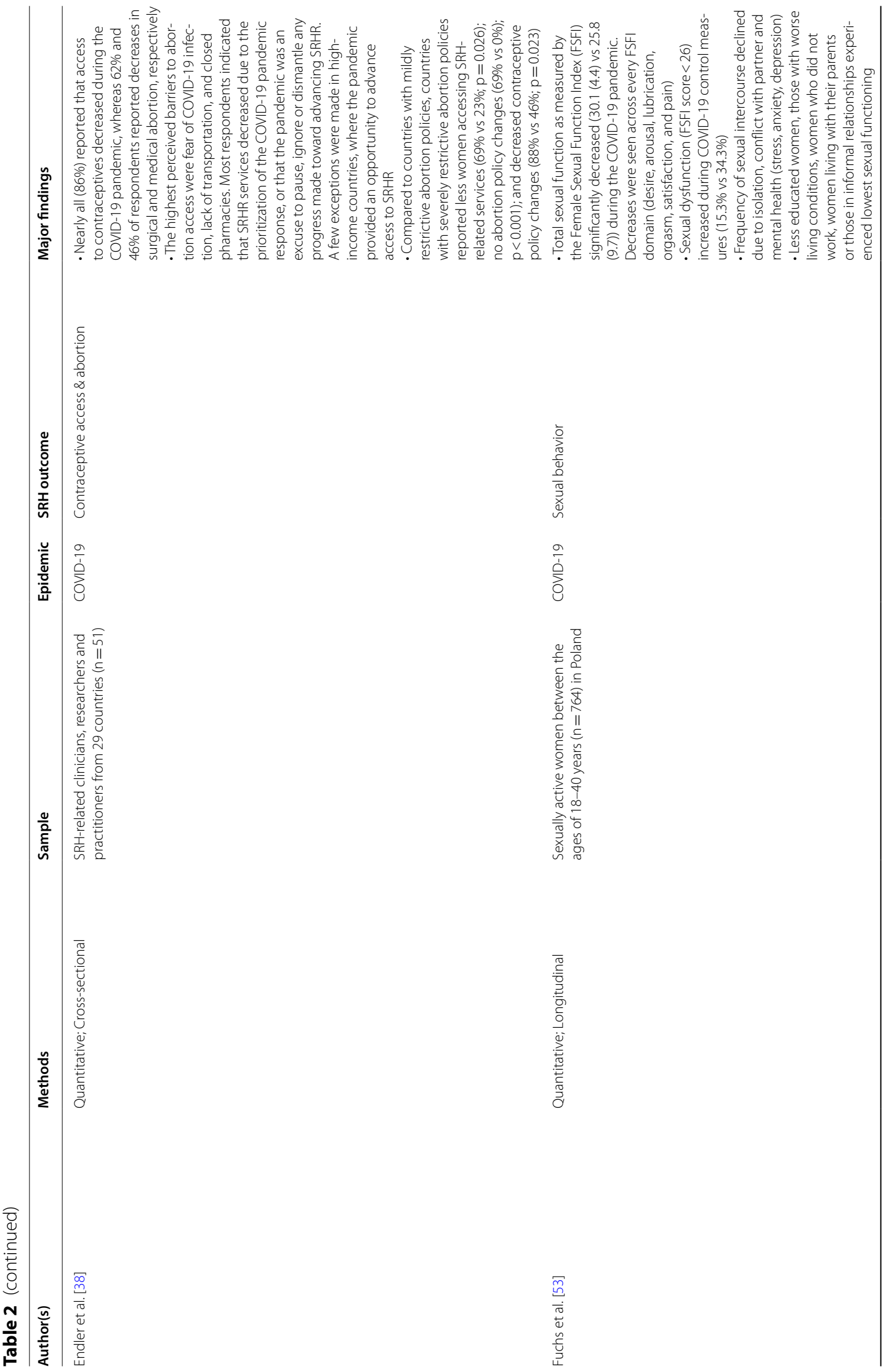




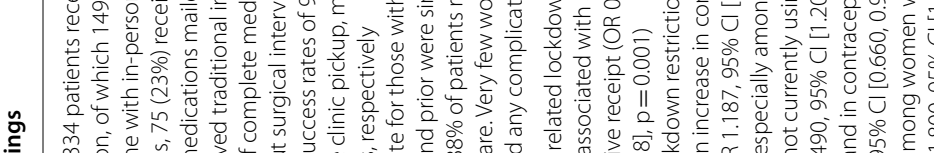

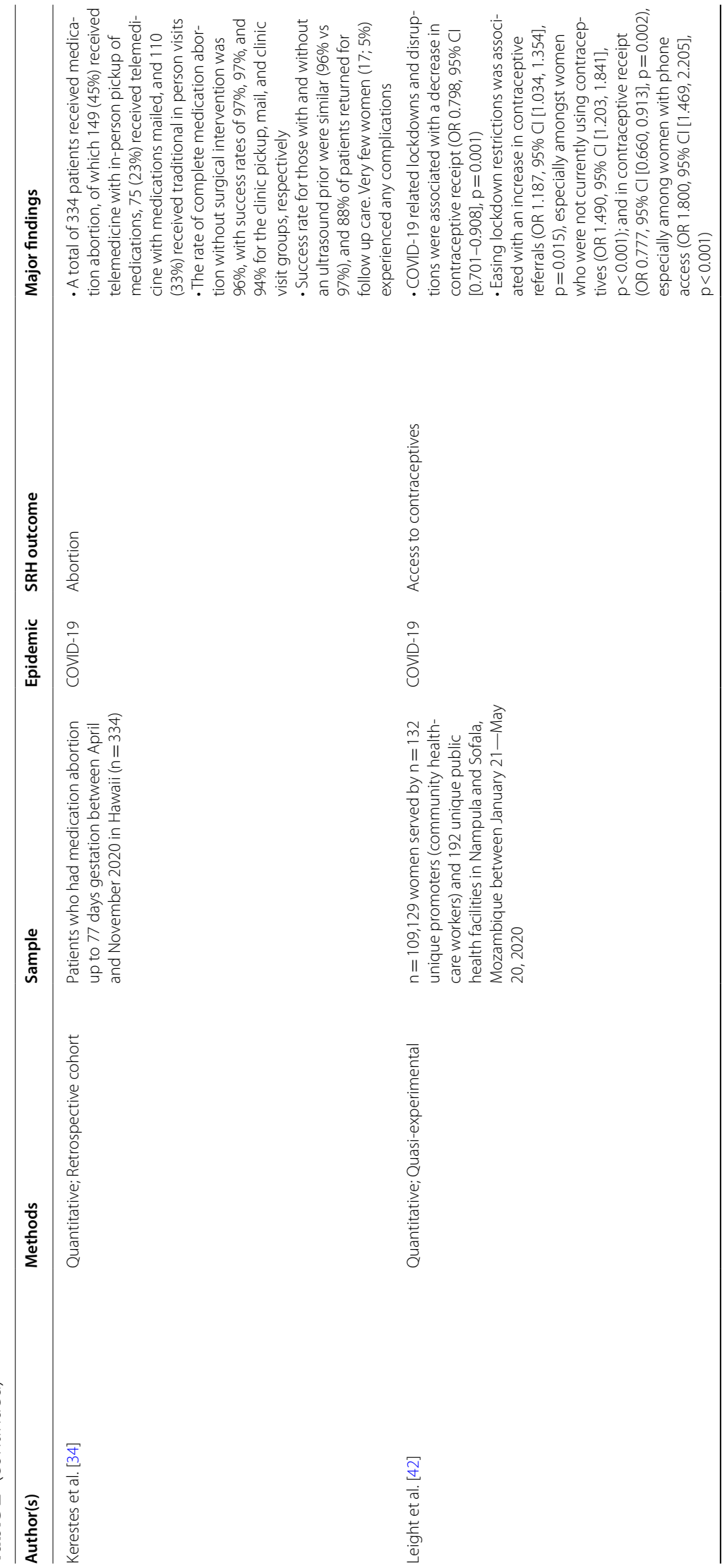




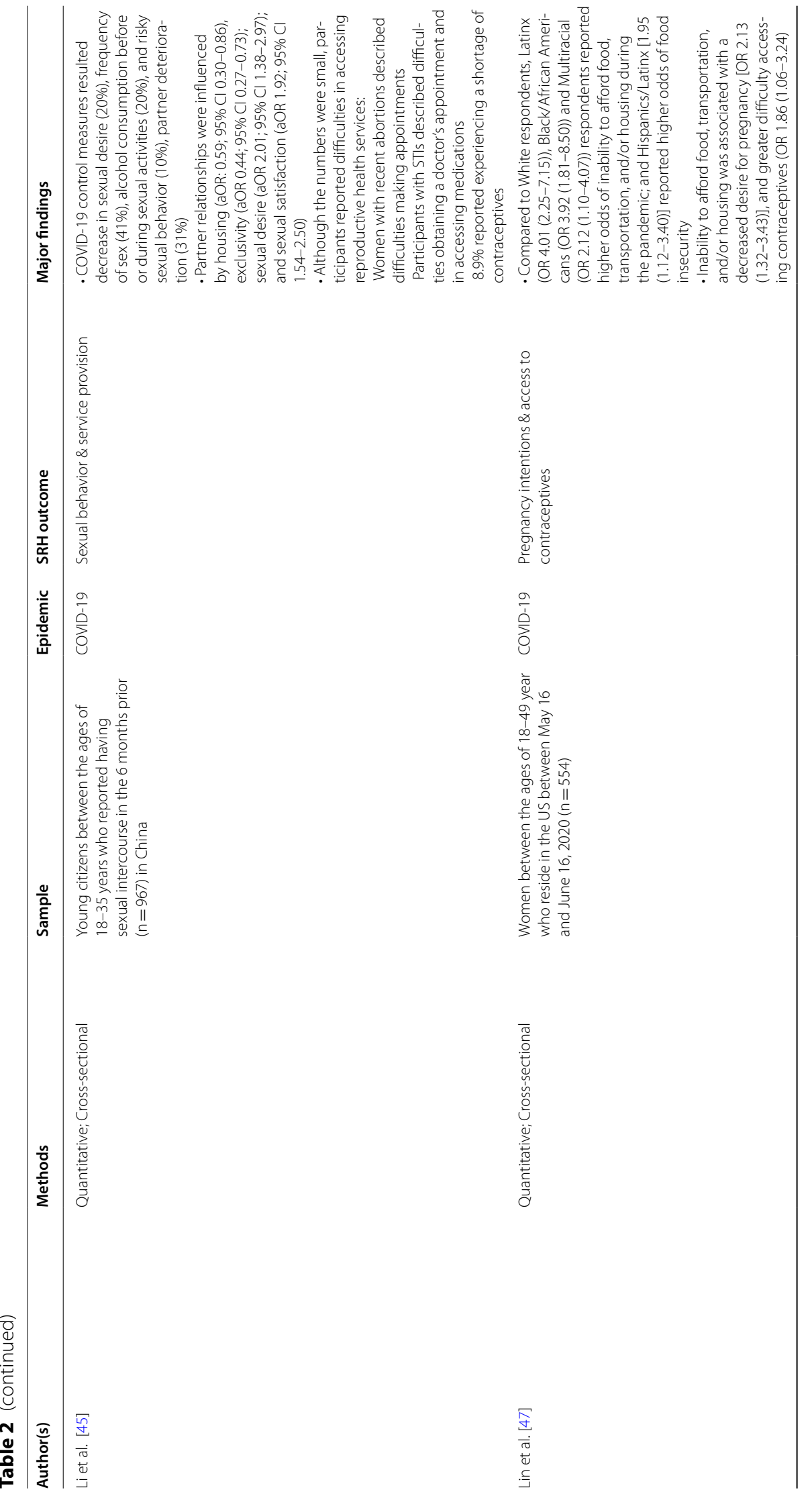


מ

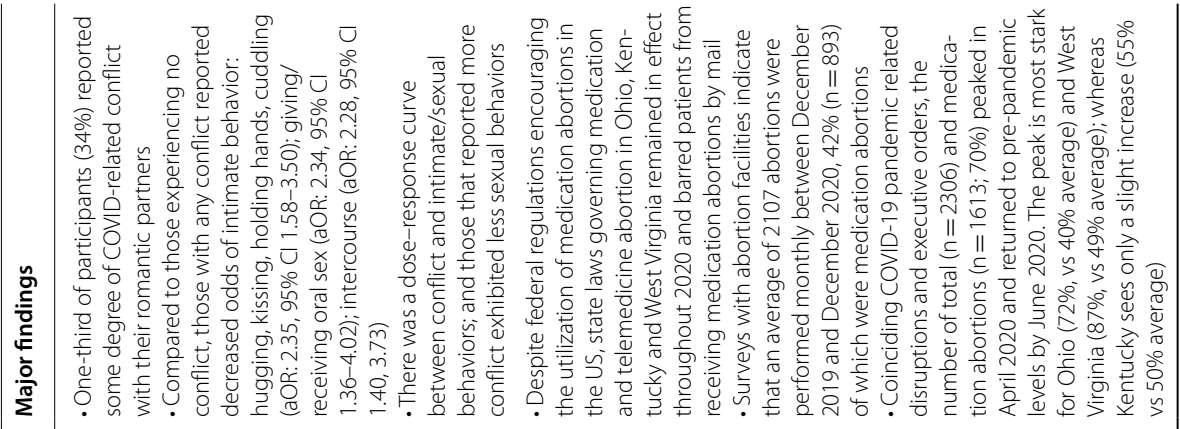

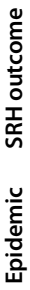

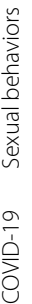

$\frac{0}{0}$
$\frac{0}{\overline{0}}$
$\frac{0}{2}$

ठे

竞

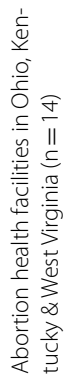

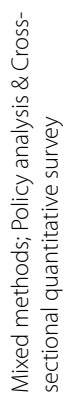

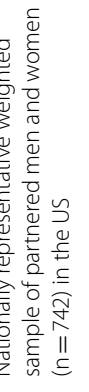




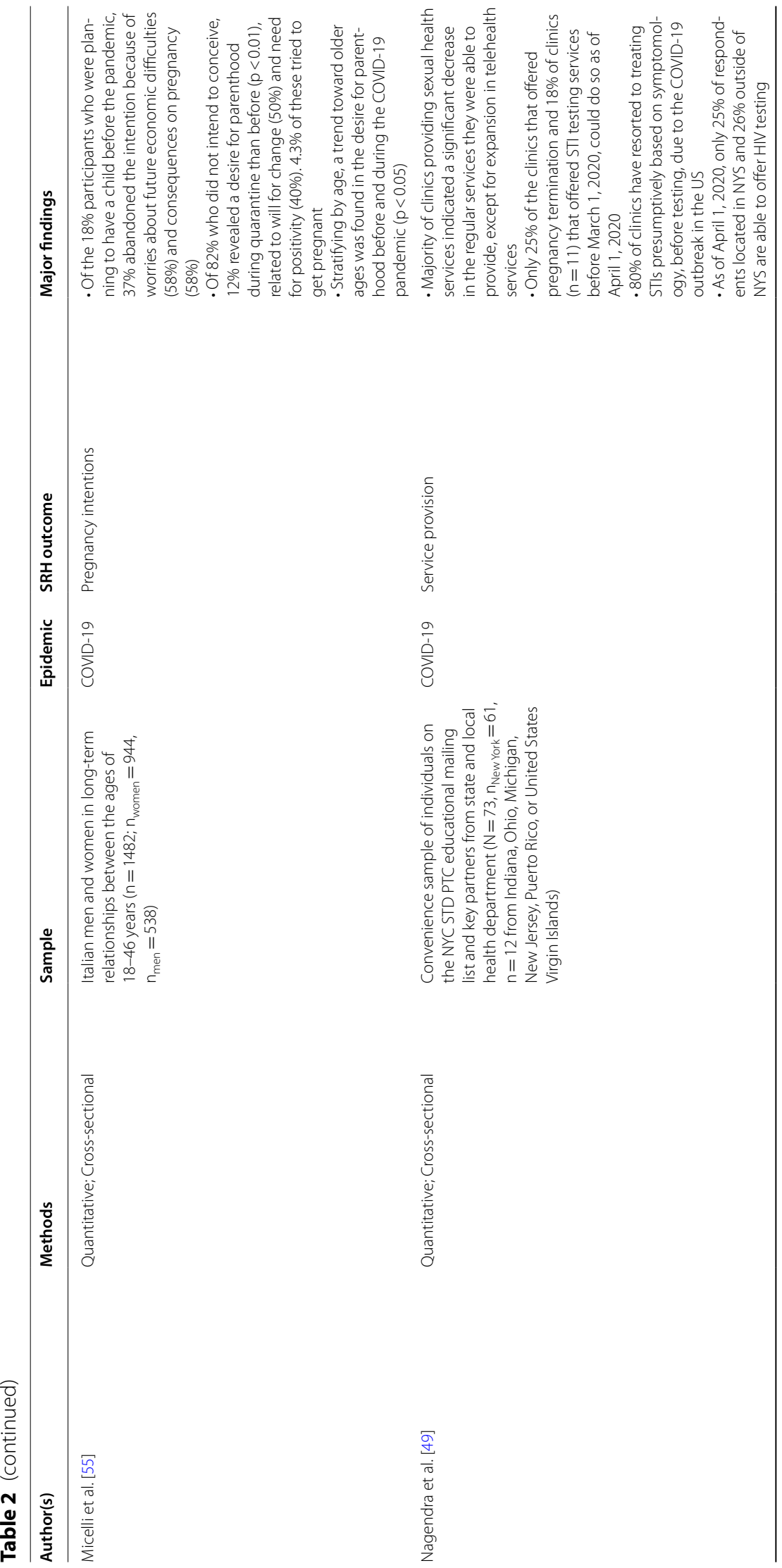




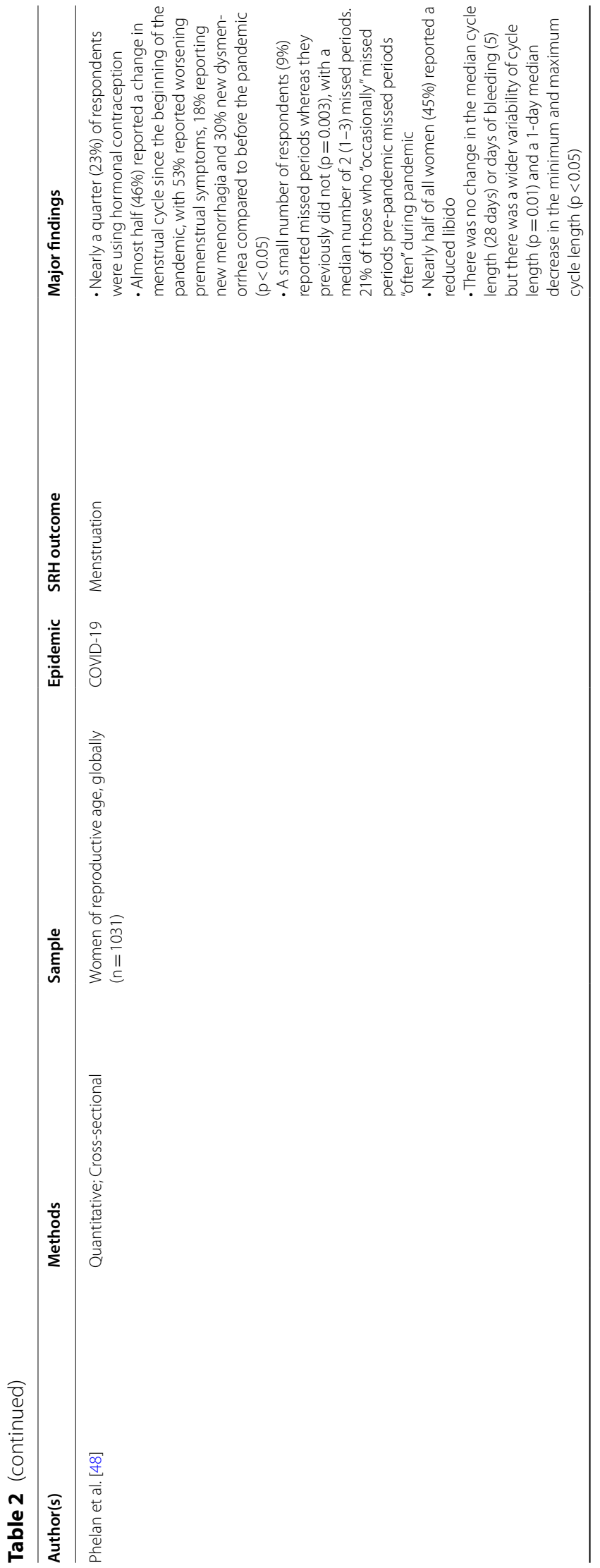




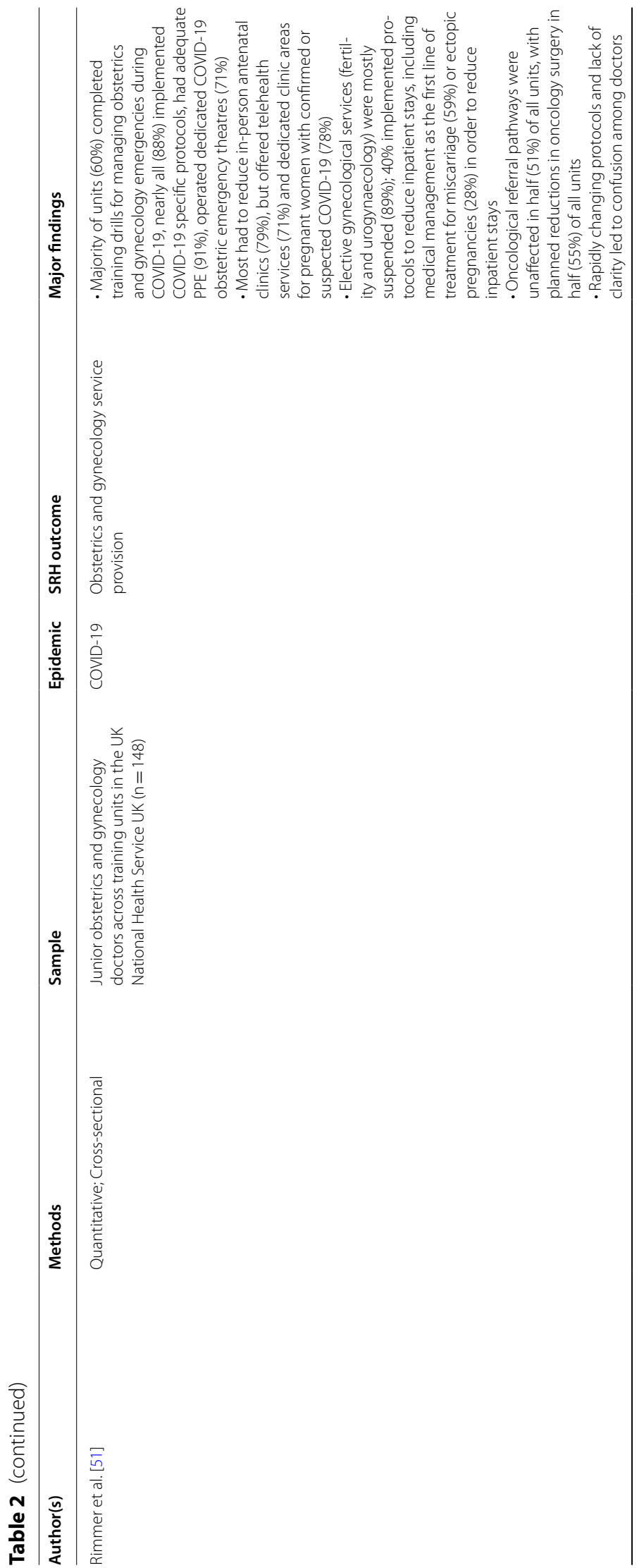




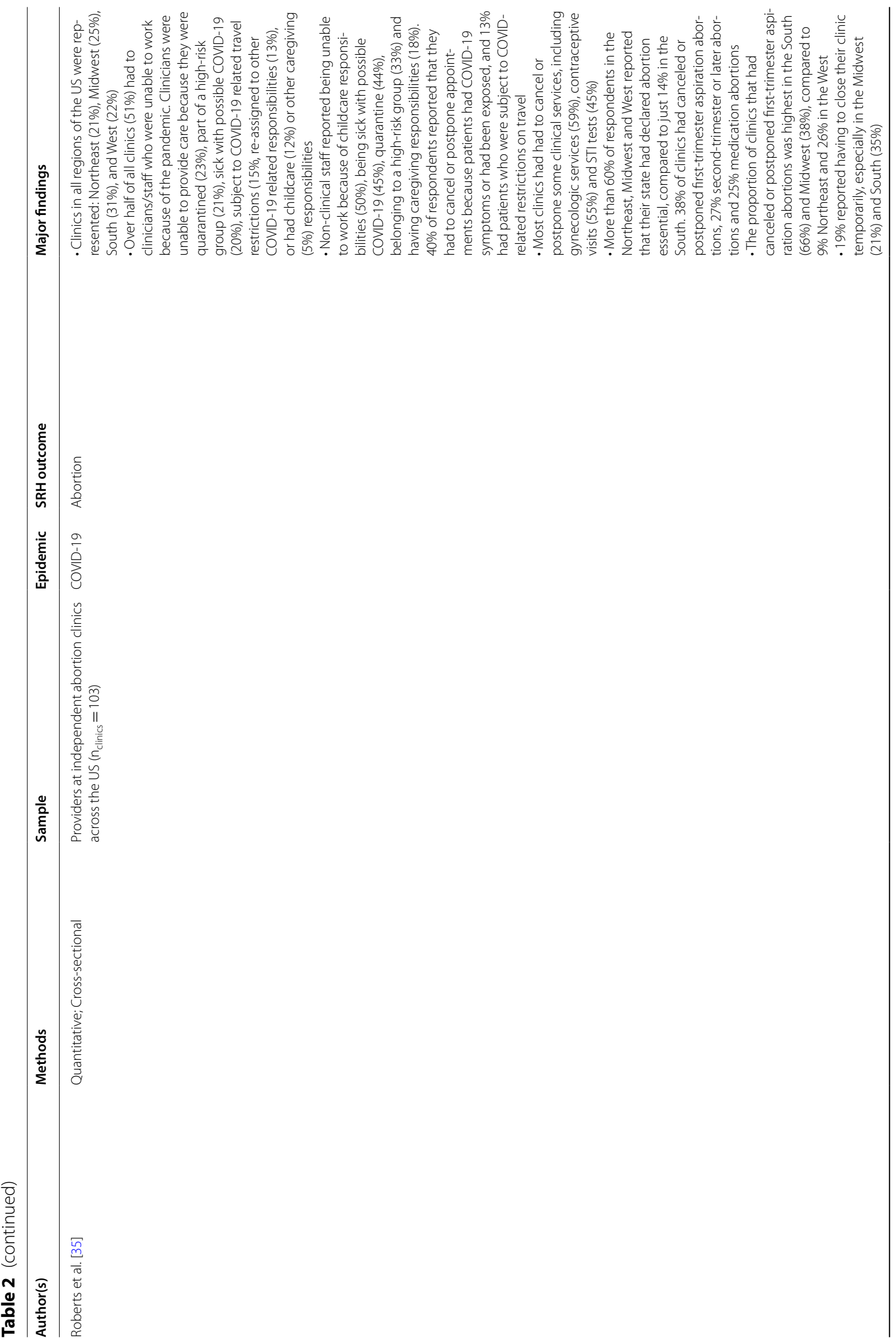




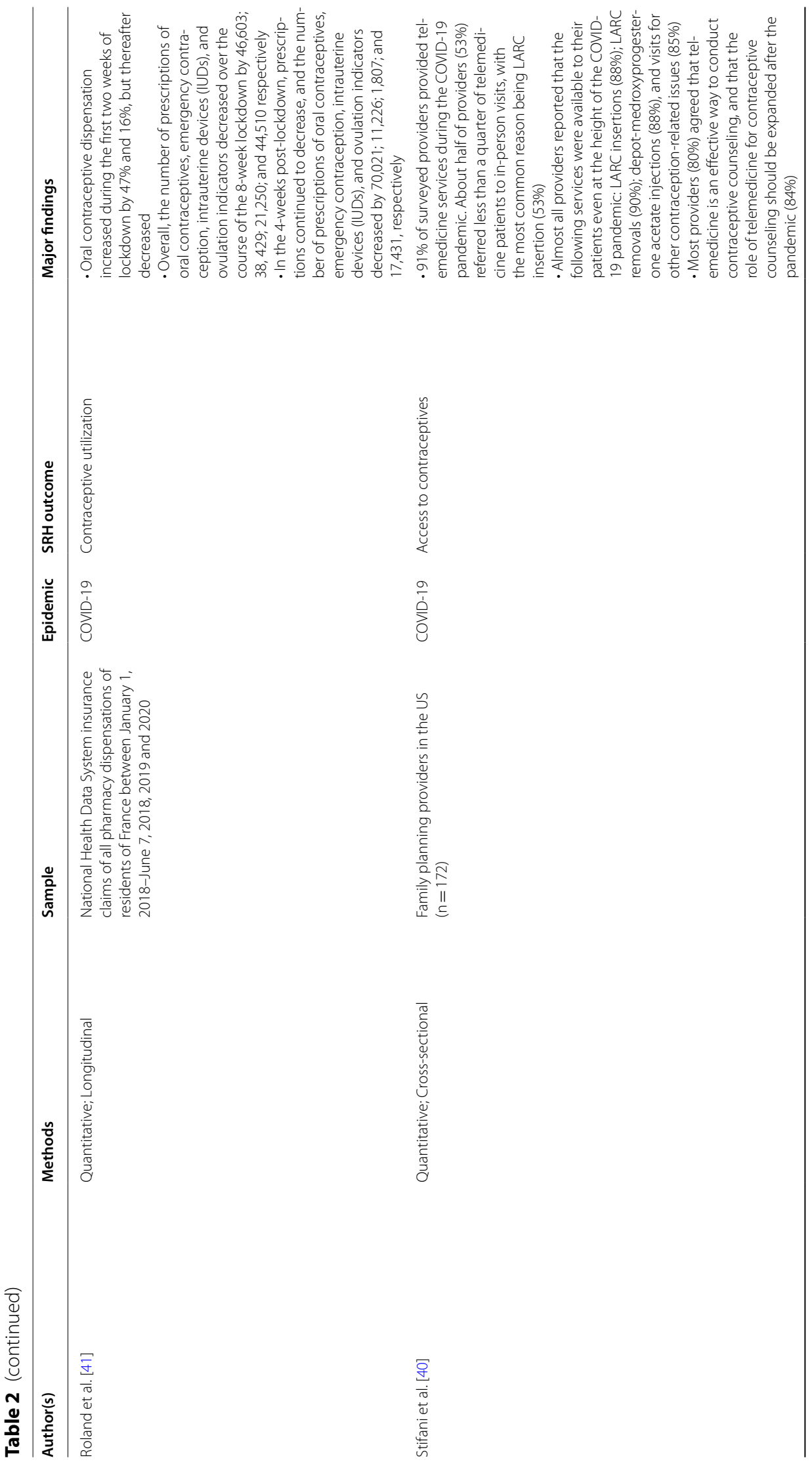




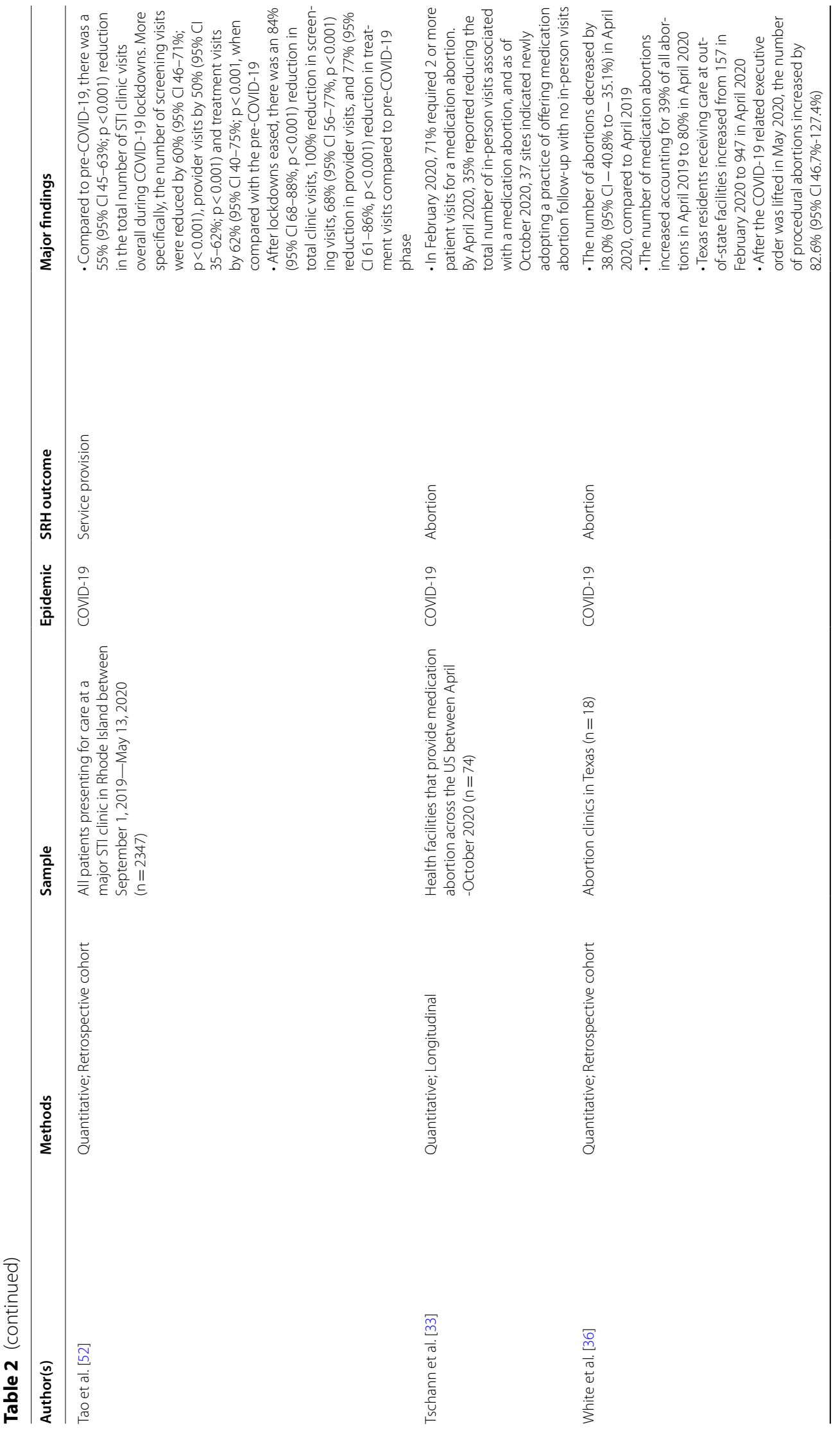




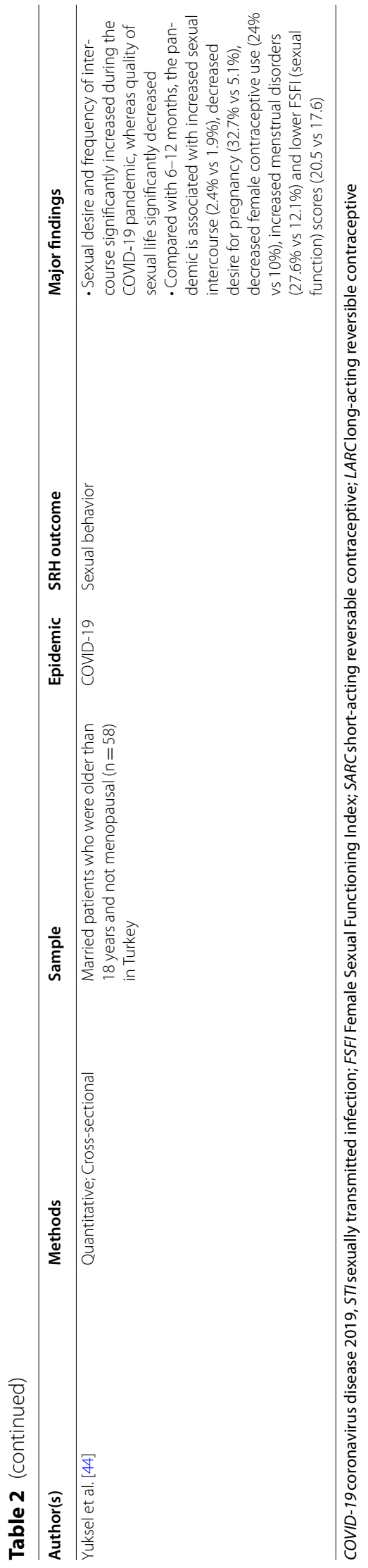




\section{Service provision}

All studies noted that COVID-19 control measures resulted in decreased service provision and/or utilization. A study of providers from sexual health clinics across the US indicated that abortion services, HIV and STI testing decreased by $76 \%, 75 \%$ and $82 \%$, respectively [49]. Consequently, telehealth services expanded, and provided greater access to services such as STI treatment based on symptomology and self-managed abortion. Using difference-in-difference analyses, Aiken et al. [32] found that requests for self-managed abortions increased during COVID-19, especially in states with greater stay-athome behaviors, restrictions on in-clinic abortions, and/ or those with especially high rates of COVID-19 incidence. Dell'Utri et al. compared obstetric and gynecological $(\mathrm{OB} / \mathrm{GYN})$ emergency service admissions during the COVID-19 pandemic, to the same period the year prior and found that overall admissions decreased by over $35 \%$ [50]. This translated to reduced admissions for complications related to pregnancy and gynecology. Similarly, Rimmer et al. (2020) reported changes to OB/GYN service provision in response to the COVID-19 pandemic in the UK [51]. These changes included reduced in-person antenatal care, elective procedures (such as fertility treatments or urogynecology), and inpatient stays. Patient-level outcomes were not reported, however. In China, women reported difficulties in accessing antenatal and/or maternal care; and obtaining appointments or medications for abortion services and STI testing [45]. Compared to pre-COVID-19 related lockdowns, the total number of clinic visits for STI screening, provider appointments and treatment decreased by $55 \%$, and $84 \%$, respectively at a STI clinic in Rhode Island during and after COVID-19 lockdown [52]. It is unclear how much of this decrease is attributed to pandemic-related lockdowns, fear of exposure, or decreased incidence of STIs due to decreased sexual activity. Disparities in service provision and/or utilization are unknown, however, as results are not described by sociodemographic status. Moreover, little is known about the impact of COVID19 on fertility treatments and gynecological cancer screenings and treatment. Only one study, from Australia, reported that several women trying to conceive had actively stopped trying or were unable to continue because their in-vitro fertilization appointments had been cancelled [46].

\section{Sexual behavior}

Several studies examined changes in sexual behaviors and functioning during the COVID-19 pandemic. Married women in Turkey reported increased sexual desire and frequency of intercourse, but lower sexual functioning and quality of sexual life based on the Female Sexual Function Index (FSFI) [44]. Fuchs et al. [53] also used the FSFI to examine sexual functioning among women of reproductive age in Poland and found that overall sexual functioning and each FSFI domain (desire, arousal, lubrication, orgasm, satisfaction, and pain) decreased significantly, and sexual dysfunction doubled. In this study, women of lower socioeconomic status experienced lowest sexual functioning. Moreover, frequency of sexual activity declined due to isolation, conflict with partners and mental health. Decreases in sexual activity also included decreases in risky sexual behaviors in China [45]. Partner conflict was explicitly examined in the US, where one-third (34\%) of all participants reported some degree of COVID-19 related conflict. An inverse doseresponse relationship was observed between relationship conflict, sexual activity and other intimate behaviors [54].

\section{Pregnancy intentions}

In examining pregnancy intentions, a study in Turkey reported a $28 \%$ decline in pregnancy desire among women of reproductive age as result of the COVID-19 pandemic [44]. In Italy, $18 \%$ of respondents intended on getting pregnant before the pandemic, however, over one-third abandoned their intention due to future economic difficulties and further straining the healthcare system. In contrast, some respondents who did not intend to conceive reported doing so because of a need for positivity [55]. In Australia, most women surveyed indicated that they were trying to avoid pregnancy, and that the pandemic had not changed their pregnancy intentions. In the US, survey respondents indicated that the pandemic exacerbated housing and food insecurity among racial/ethnic minorities, which was associated with a decreased desire for pregnancy by over twofold [47].

\section{Risk of bias}

Overall studies were of low quality with scores ranging from 13 to $37(40-88 \%)$ and averaging $23(56 \%)$ points across all 24 eligible studies (Table 3 ). Of the 16 QATSDD items, the highest scoring items were a specific statement of aims/objectives (item 2); a clear description of the research setting (item 3), and fit between research question and analysis method (item 12). On average, lowest scoring items included the use of an explicit theoretical framework (item 1), statistical assessment of reliability and validity of measurement tool(s) (item 9), and evidence of user involvement in design (e.g., pilot study, informed by persons with lived experience, etc.; item 15). Although theoretical frameworks were not explicitly included, most authors stated why their research question was important within their given context. Items that 


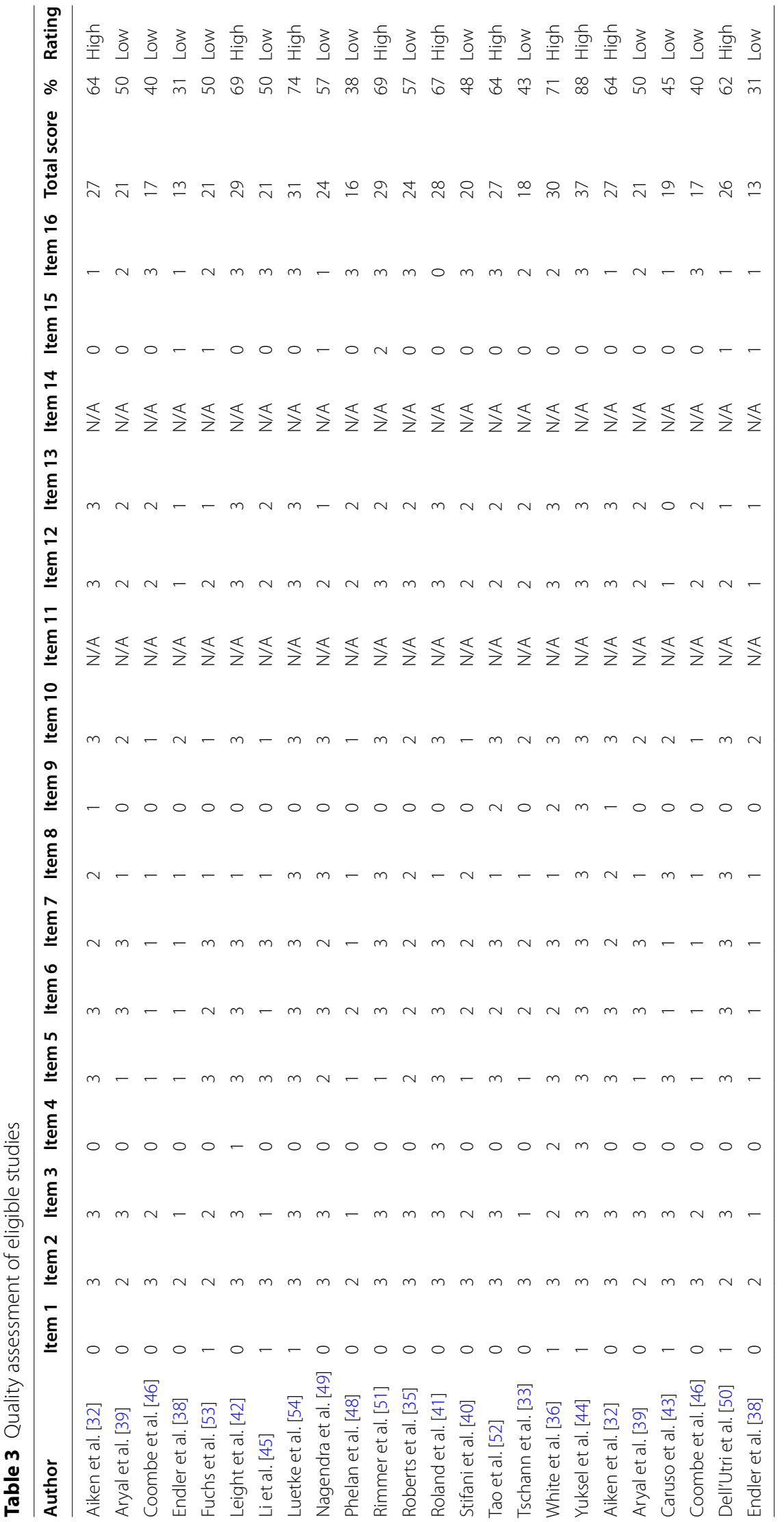




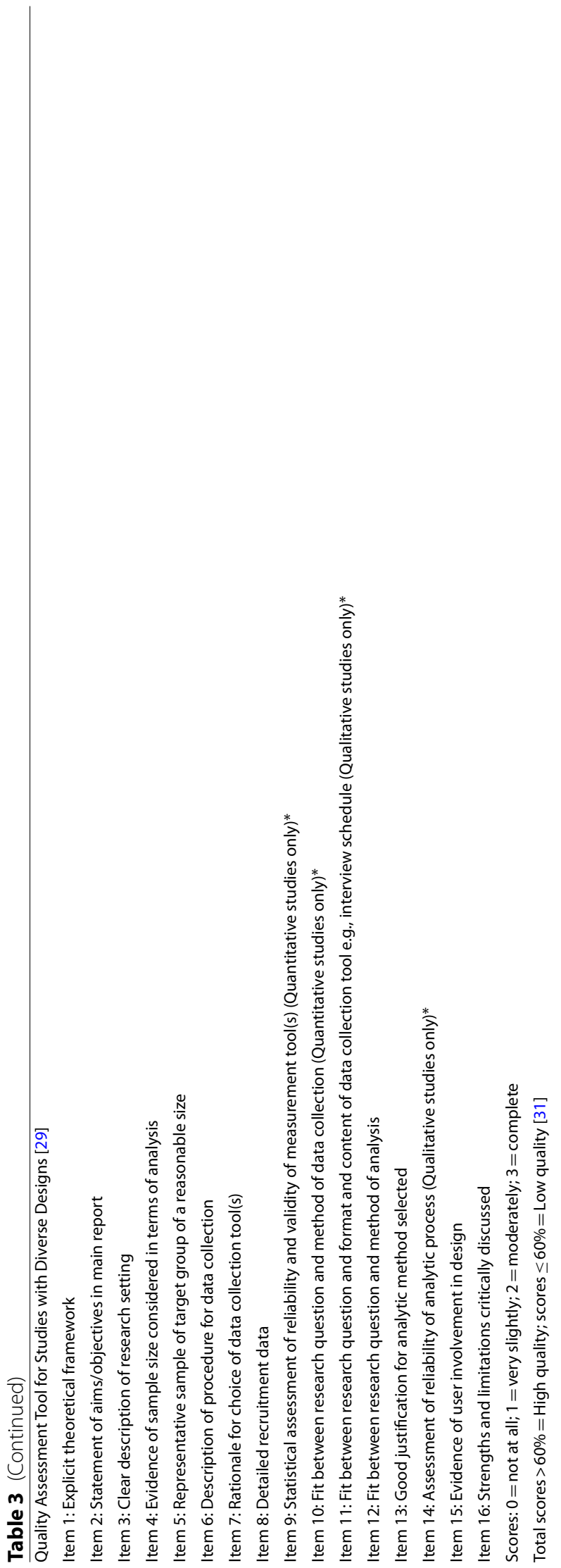


did not score well may have been due to study design (e.g., observational vs randomized control trials). Similarly, statistical assessment of reliability and validity of measurement tool(s) may not have been considered due to lack of time for test re-test sampling, or the lack of validated tools measuring SRH-related outcomes. Finally, user involvement may not have been feasible or ethical during an epidemic.

\section{Discussion}

Beyond COVID-19 morbidity and mortality, all women and girls, including underserved populations, racial/ ethnic or sexual minorities, immigrants and those with intersectional identities, will experience immediate and long-term consequences to their sexual and reproductive health $[2,12,13]$. Results from this review suggest that the indirect impact of the COVID-19 pandemic on SRH include significant reductions in access to abortion, contraceptives, and OB/GYN service provision. All studies reported in this review indicate that the total number of abortions decreased during the pandemic, but it is not clear whether this is due to decreased access because of pandemic-related lockdowns and SRH not being deemed an essential service, or due to decreases in sexual activity and changes in pregnancy intentions, as reported by some studies. Among women receiving abortions, the number of medication abortions increased, whereas surgical abortions decreased. Studies reported innovations such as telemedicine with or without in-person follow up visits for medication abortions, which were deemed safe, accessible and without complications [56]. The studies include in this review did not examine abortion access by sociodemographic characteristic or socioeconomic status, and disparities or inequities are unknown. The lack of unified abortion or epidemic control policies in response to COVID-19, however, likely widened existing health inequities [57].

Based on this review, COVID-19 pandemic related disruptions to family planning services were reported to decrease access to contraceptives, prescriptions, and/ or uptake globally. This may be an unintended consequence of prioritizing COVID-19 response over SRH needs, and it is unclear how changes in sexual behavior and pregnancy intentions impact contraception uptake. Most studies included in this review did not report contraception method. The limited number of studies that did report contraception method suggest that service disruptions disproportionately impact women who rely on SARCs, as LARCS have been proven to be effective past their intended duration [58]. Of concern is that the reduction in contraceptive use was sustained, even once lockdowns were eased in some places [41]. On the other hand, some places showed a promising rebound, especially among women who were not using contraceptives previously and women of lower socio-economic status [42]. Only one study examined how poverty related factors are negatively associated with contraceptive access in the US [47].

Our search returned no studies on the impact of other respiratory epidemics on women and girls SRH outcomes (not including pregnancy and birth-related outcomes, gender-based violence, and maternal and child health). This may be because the prioritization of epidemic response has overshadowed SRH, the historic lack of investment in SRH, or the dismissal of SRH as rooted in structural gender inequities. However, given the rise in emerging infectious diseases [59] and increasing calls for attention to SRH during pandemics/epidemics [2, 12, 13], this finding emphasizes the need to examine the full range of SRH outcomes, that is inclusive of HIV/STIs; comprehensive sexuality education; safe abortion; prevention, detection, and counselling for gender-based violence; prevention, screening and treatment of infertility and gynecological cancers; and counseling and care for sexual health and well-being [60]. High quality evidence of the indirect, downstream consequences of epidemics is needed to inform future policy and planning, ensure SRH equity, and generate equitable access to the full range of SRH services. Moreover, few studies included in this review examined the indirect impact of COVID-19 and the pandemic response on SRH outcomes among underserved populations, racial/ethnic or sexual minorities, immigrants, or those with intersectional identities. The pandemic has been found to exacerbate poverty, disproportionately impact people of lower socioeconomic status, and aggravate existing health issues, including those related to SRH [61]. Interventions are critically needed to sustain adequate access to abortion, family planning, STI/HIV testing and treatment, ensure continuity of fertility treatments, gynecological cancer screenings and treatment, and other SRH service provision, especially among women of lower socioeconomic status, to reduce the number of unintended pregnancies, unsafe abortions, STI/HIV transmission, and halt the decades of progress made on health and development [62].

$\mathrm{SRH}$ is a human right that is vital for sustainable development, and should be among the least restrictive solutions in the context of epidemic control [2, 13, 63]. Historically, restrictive SRH policies have perpetuated inequities among Black, Latinx, and immigrant women, and are expected to widen as a result of COVID-19 and related policies for epidemic control. Few studies of respiratory epidemics and SRH explicitly examined outcomes among women with diverse lived experiences, despite the accumulating evidence that indicate that COVID-19 disproportionately impacts racial/ethnic 
minorities, immigrants, and people with lower socioeconomic status [12, 64]. This review highlights a gap in research of SRH service provision, access and utilization among marginalized groups of women and girls and those with intersectional identities, including adolescent and young girls, those with disabilities, sexual or ethnic/racial minorities, refugees and immigrants, many of whom experience difficulties in accessing SRH services notwithstanding an epidemic $[10,11,13]$. Several studies noted the expansion of telehealth services, offers an opportunity to reach more women and girls, including those traditionally underserved. Yet, little research on how telehealth has improved or constrained SRH access for underserved populations has been published. This review highlights the need to understand the indirect impact of COVID-19 and its control measures on the wider range of SRH outcomes and populations of women and girls in the long-term.

This study is not without limitations. Given the rapid timeline of this review and the constantly evolving research on COVID-19, we included readily available studies on COVID-19 impacts on SRH at the time of review, but new findings emerge on a weekly basis. Moreover, there were no restrictions on location, and generalizability of results may be inadequate due to variations in epidemic control policies. Non-respiratory epidemics (e.g., Ebola, HIV, Zika, etc.) have also impacted SRH outcomes; however, these were not included as modes of transmission and infection control measures varied too greatly. Although we apply a reproductive justice lens, we excluded maternal and child health outcomes beyond pregnancy, childbirth and violence. While these are an important aspect of reproductive justice, this literature seemed to be its own body of work and dedicated systematic reviews have been published elsewhere [17-28]. Finally, the majority of studies included in this review were of low quality; possibly because the majority of studies reported in this review were observational, and more rigorous research on the impact of pandemics on SRH is needed. Randomized-control trials are the gold-standard for high-quality studies, however, they are not always feasible, practical or ethical within the context of an infectious disease epidemic, and studies without a comparison group should be interpreted with caution. Conversely, quasi-experimental designs are useful in determining causal relationships when randomized control trials cannot be used for practical or ethical reasons [65]. The COVID-19 pandemic provides an opportunity to use quasi-experimental designs to better understand the indirect impact of COVID-19 and the pandemic response on SRH outcomes among marginalized women and girls. Future research using quasi-experimental designs are needed to provide robust evidence of the impact of interventions and/or policy changes (e.g., telemedicine with in-person versus mail order medication abortion, executive orders that did not deem SRH as an essential service, etc.) on SRH-related outcomes.

By being aware of the impacts of COVID-19 on SRH, policy makers can be better prepared to enact guidelines and policies that promote reproductive justice and access to equitable, timely SRH, despite lockdowns. Given the service disruptions evident in this review, providers should prioritize education and provision of various contraceptive methods, and when appropriate, should counsel and allow patients to consider switching methods. Patient education on the range of contraceptive methods, protocols for switching methods, at-home use of contraceptive methods (including injectables [66]) and self-managed abortion can be completed via telehealth, which may provide an opportunity to reach more women and girls. Although not included in this review, COVID19 has resulted in notable increases in gender-based violence and reproductive coercion [28, 67], making access to contraceptives and abortion services vital for ensuring access to care and reproductive justice.

Changes to service provision, in response to COVID19 , must consider historical inequities in access to SRH services. Clear and consistent guidelines for changes to service provision that ensure access to quality SRH services are needed. Moreover, increased efforts should be made to collect sociodemographic information to better understand the indirect and downstream impact of the COVID-19 pandemic on SRH for diverse groups. Finally, while the expansion of telehealth services provides an opportunity to reach underserved populations, privacy concerns, disparities in access to technology, and longstanding impacts of racism on care uptake must also be considered.

\section{Conclusion}

As COVID-19 presents new challenges to accessing essential SRH services, the application of a reproductive justice lens is crucial to ensure SRH inequities do not continue to widen. Evidence suggests that COVID19 , and its control measures disproportionately impact women's SRH outcomes. Results indicate that OB/GYN and SRH service provision, pregnancy intentions and sexual behavior, access to family planning, contraceptives and abortion markedly decreased, as an indirect consequence of the COVID-19 pandemic response. Accumulating evidence indicates that COVID-19 disproportionately impacts marginalized and underserved populations directly, yet these are the groups least represented in the research. More research dedicated to the diverse lived experiences of women and higher quality evidence is needed to prevent and mitigate the indirect 


\section{impact of COVID-19 and lockdown measures on long- term SRH outcomes.}

\section{Acknowledgements}

Not applicable.

\section{Authors' contributions}

Title, abstract, and full text screening of eligible articles were completed by TM, AK, AD, and GS. Study data (author, study type, epidemic, SRH outcome and major findings) were abstracted and risk of bias was assessed by AK and TM. GS conceptualized the paper. All authors read and approved the final manuscript.

\section{Funding}

Research reported in this publication was supported by the Eunice Kennedy Shriver National Institute of Child Health and Human Development under grant number P2CHD058486, awarded to the Columbia Population Research Center. The content is solely the responsibility of the authors and does not necessarily represent the official views of the National Institutes of Health. No authors report any conflicts of interest.

\section{Availability of data and materials}

Not applicable.

\section{Declarations}

\section{Ethics approval and consent to participate}

Not applicable.

\section{Consent for publication}

Not applicable.

\section{Competing interests}

The authors declare that they have no competing interests.

\section{Author details}

${ }^{1}$ Department of Epidemiology, Mailman School of Public Health, Columbia University, New York, NY, USA. ${ }^{2}$ Department of Community Health Sciences, Fielding School of Public Health, University of California Los Angeles, Los Angeles, CA, USA. ${ }^{3}$ Social Intervention Group, School of Social Work, Columbia University, New York, NY, USA. ${ }^{4}$ Heilbrunn Department of Population and Family Health, Mailman School of Public Health, Columbia University, New York, NY, USA.

Received: 2 February 2021 Accepted: 6 November 2021 Published online: 20 December 2021

\section{References}

1. Cousins S. COVID-19 has "devastating" effect on women and girls. Lancet. 2020;396(10247):301-2.

2. Hall KS, Samari G, Garbers S, Casey SE, Diallo DD, Orcutt M, et al. Centring sexual and reproductive health and justice in the global COVID-19 response. Lancet. 2020;395(10231):1175-7.

3. Wenham C, Smith J, Morgan R. COVID-19: the gendered impacts of the outbreak. Lancet. 2020;395(10227):846-8.

4. Tang K, Gaoshan J, Ahonsi B, Ali M, Bonet M, Broutet N, et al. Sexual and reproductive health $(\mathrm{SRH})$ : a key issue in the emergency response to the coronavirus disease (COVID- 19) outbreak. Reprod Health. 2020;17(1):59.

5. Hussein J. COVID-19: What implications for sexual and reproductive health and rights globally? Sex Reprod Health Matters. 2020;28(1):1746065.

6. Church K, Gassner J, Elliott M. Reproductive health under Covid-19challenges of responding in a global crisis. Sex Reprod Health Matters. 2020:28:1-3.

7. Tran NT, Tappis H, Spilotros N, Krause S, Knaster S. Not a luxury: a call to maintain sexual and reproductive health in humanitarian and fragile settings during the COVID-19 pandemic. Lancet Glob Health. 2020;8(6):e760-1.

8. McGinn T. Reproductive health of war-affected populations: what do we know? Int Fam Plan Perspect. 2000;26(4):174-80.

9. Ludmir J, Fabi RE. Pregnancy, pandemics, and public health policy: the disparate impact of COVID-19 on pregnant immigrants. Womens Health Issues. 2020:31:195-7.

10. Hall KS, Moreau C, Trussell J. Determinants of and disparities in reproductive health service use among adolescent and young adult women in the United States, 2002-2008. Am J Public Health. 2012;102(2):359-67.

11. Murray Horwitz ME, Pace LE, Ross-Degnan D. Trends and disparities in sexual and reproductive health behaviors and service use among young adult women (aged 18-25 years) in the United States, 2002-2015. Am J Public Health. 2018;108(S4):S336-43.

12. Desai S, Samari G. COVID-19 and immigrants' access to sexual and reproductive health services in the United States. Perspect Sex Reprod Health. 2020. https://doi.org/10.1363/psrh.12150.

13. Maier M, Samari G, Ostrowski J, Bencomo C, McGovern T. 'Scrambling to figure out what to do': a mixed method analysis of COVID-19's impact on sexual and reproductive health and rights in the United States. BMJ Sex Reprod Health. 2021;47(4):e16.

14. Riley T, Sully E, Ahmed Z, Biddlecom A. Estimates of the potential impact of the COVID-19 pandemic on sexual and reproductive health in low-and middle-income countries. Int Perspect Sex Reprod Health. 2020:46:46.

15. Bond TM. Reproductive justice and women of color. Retrieved from A Primer On Reproductive Justice And Social Change; 2007

16. Ross L. What is reproductive justice? Retrieved from A Primer On Reproductive Justice And Social Change; 2007

17. Chmielewska B, Barratt I, Townsend R, Kalafat E, van der Meulen J, Gurol-Urganci I, et al. Effects of the COVID-19 pandemic on maternal and perinatal outcomes: a systematic review and meta-analysis. Lancet Global Health. 2021. https://doi.org/10.1016/S2214-109X(21)00079-6.

18. Wei SQ, Bilodeau-Bertrand M, Liu S, Auger N. The impact of COVID-19 on pregnancy outcomes: a systematic review and meta-analysis. CMAJ. 2021:193(16):E540-8

19. Gajbhiye RK, Modi DN, Mahale SD. Pregnancy outcomes, newborn complications and maternal-fetal transmission of SARS-CoV-2 in women with COVID-19: a systematic review of 441 cases. MedRxiv. 2020. https://doi. org/10.1101/2020.04.11.20062356.

20. Dubey P, Reddy S, Manuel S, Dwivedi AK. Maternal and neonatal charac teristics and outcomes among COVID-19 infected women: an updated systematic review and meta-analysis. Eur J Obstet Gynecol Reprod Biol. 2020;252:490-501.

21. Smith V, Seo D, Warty R, Payne O, Salih M, Chin KL, et al. Maternal and neonatal outcomes associated with COVID-19 infection: a systematic review. PLOS ONE. 2020;15(6):e0234187.

22. Piquero AR, Jennings WG, Jemison E, Kaukinen C, Knaul FM. Evidence from a systematic review and meta-analysis: domestic violence during the COVID-19 Pandemic. J Crim Justice. 2021:74:101806.

23. Hessami K, Romanelli C, Chiurazzi M, Cozzolino M. COVID-19 pandemic and maternal mental health: a systematic review and meta-analysis. J Maternal-Fetal Neonatal Med. 2020;1:1-8.

24. Kotlar B, Gerson E, Petrillo S, Langer A, Tiemeier H. The impact of the COVID-19 pandemic on maternal and perinatal health: a scoping review. Reprod Health. 2021;18(1):1-39.

25. Yang Z, Wang M, Zhu Z, Liu Y. Coronavirus disease 2019 (COVID-19) and pregnancy: a systematic review. J Matern Fetal Neonatal Med. $2019 \cdot 2020 \cdot 1-4$

26. Allotey J, Stallings E, Bonet M, Yap M, Chatterjee S, Kew T, et al. Clinica manifestations, risk factors, and maternal and perinatal outcomes of coronavirus disease 2019 in pregnancy: living systematic review and meta-analysis. BMJ. 2020;370:m3320.

27. Mittal S, Singh T. Gender-based violence during COVID-19 pandemic: a mini-review. Front Glob Womens Health. 2020;1:4.

28. Sánchez OR, Vale DB, Rodrigues L, Surita FG. Violence against women during the COVID-19 pandemic: an integrative review. Int J Gynecol Obstet. 2020;151(2):180-7.

29. Sirriyeh R, Lawton R, Gardner P, Armitage G. Reviewing studies with diverse designs: the development and evaluation of a new tool. J Eval Clin Pract. 2012;18(4):746-52. 
30. Fenton L, Lauckner H, Gilbert R. The QATSDD critical appraisal tool: comments and critiques. J Eval Clin Pract. 2015;21(6):1125-8.

31. Mcllveen R, Curran D, Mitchell R, DePrince A, O'Donnell K, Hanna D. A meta-analytic review of the association between alienation appraisals and posttraumatic stress disorder symptoms in trauma-exposed adults. J Trauma Stress. 2020;33(5):720-30.

32. Aiken ARA, Starling JE, Gomperts R, Tec M, Scott JG, Aiken CE. Demand for self-managed online telemedicine abortion in the United States during the coronavirus disease 2019 (COVID-19) pandemic. Obstet Gynecol. 2020;136(4):835-7.

33. Tschann M, Ly ES, Hilliard S, Lange HLH. Changes to medication abortion clinical practices in response to the COVID-19 pandemic. Contraception. 2021;104(1):77-81.

34. Kerestes C, Murayama S, Tyson J, Natavio M, Seamon E, Raidoo S, et al. Provision of medication abortion in Hawai'i during COVID-19: practical experience with multiple care delivery models. Contraception. 2021;104(1):49-53.

35. Roberts SCM, Schroeder R, Joffe C. COVID-19 and independent abortion providers: findings from a rapid-response survey. Perspect Sex Reprod Health. 2020:52(4):217-25.

36. White K, Kumar B, Goyal V, Wallace R, Roberts SCM, Grossman D. Changes in abortion in Texas following an executive order ban during the coronavirus pandemic. JAMA. 2021;325(7):691-3.

37. Mello K, Smith MH, Hill BJ, Chakraborty P, Rivlin K, Bessett D, et al. Federal, state, and institutional barriers to the expansion of medication and telemedicine abortion services in Ohio, Kentucky, and West Virginia during the COVID-19 pandemic. Contraception. 2021;104(1):111-6.

38. Endler M, Al-Haidari T, Benedetto C, Chowdhury S, Christilaw J, El Kak F, et al. How the coronavirus disease 2019 pandemic is impacting sexual and reproductive health and rights and response: results from a global survey of providers, researchers, and policy-makers. Acta Obstet Gynecol Scand. 2021;100(4):571-8.

39. Aryal S, Nepal S, Ballav Pant S. Safe abortion services during the COVID -19 pandemic: a cross-sectional study from a tertiary center in Nepal. F1000Res. 2021:10:112.

40. Stifani BM, Avila K, Levi EE. Telemedicine for contraceptive counseling: an exploratory survey of US family planning providers following rapid adoption of services during the COVID-19 pandemic. Contraception. 2021;103(3):157-62.

41. Roland N, Drouin J, Desplas D, Cuenot F, Dray-Spira R, Weill A, et al. Effects of the coronavirus disease 2019 (COVID-19) lockdown on the use of contraceptives and ovulation inductors in France. Obstet Gynecol. 2021;137(3):415.

42. Leight J, Hensly C, Chissano M, Ali L. Short-term effects of the COVID19 state of emergency on contraceptive access and utilization in Mozambique. PLoS ONE. 2021;16(3):e0249195.

43. Caruso S, Rapisarda AMC, Minona P. Sexual activity and contraceptive use during social distancing and self-isolation in the COVID-19 pandemic. Eur J Contracept Reprod Health Care. 2020;25(6):445-8.

44. Yuksel B, Ozgor F. Effect of the COVID-19 pandemic on female sexual behavior. Int J Gynecol Obstet. 2020;150(1):98-102.

45. Li G, Tang D, Song B, Wang C, Qunshan S, Xu C, et al. Impact of the COVID-19 pandemic on partner relationships and sexual and reproductive health: cross-sectional, online survey study. J Med Internet Res. 2020;22(8):e20961.

46. Coombe J, Kong F, Bittleston H, Williams H, Tomnay J, Vaisey A, et al. Contraceptive use and pregnancy plans among women of reproductive age during the first Australian COVID-19 lockdown: findings from an online survey. Eur J Contracept Reprod Health Care. 2021;26(4):265-71

47. Lin TK, Law R, Beaman J, Foster DG. The impact of the COVID-19 pandemic on economic security and pregnancy intentions among people at risk of pregnancy. Contraception. 2021;103(6):380-5.

48. Phelan N, Behan LA, Owens L. The impact of the COVID-19 pandemic on women's reproductive health. Front Endocrinol (Lausanne). 2021:12:642755.

49. Nagendra G, Carnevale C, Neu N, Cohall A, Zucker J. The potential impact and availability of sexual health services during the COVID-19 pandemic. Sex Transm Dis. 2020;47(7):434-6.

50. Dell'Utri C, Manzoni E, Cipriani S, Spizzico C, Dell'Acqua A, Barbara $\mathrm{G}$, et al. Effects of SARS Cov-2 epidemic on the obstetrical and gynecological emergency service accesses. What happened and what shall we expect now? Eur J Obstet Gynecol Reprod Biol. 2020;254:64-8.

51. Rimmer MP, Al Wattar BH, Members U. Provision of obstetrics and gynaecology services during the COVID-19 pandemic: a survey of junior doctors in the UK National Health Service. BJOG. 2020;127(9):1123-8.

52. Tao J, Napoleon SC, Maynard MA, Almonte A, Silva E, Toma E, et al. Impact of the COVID-19 pandemic on sexually transmitted infection clinic visits. SexTransm Dis. 2021:48(1):e5-7.

53. Fuchs A, Matonog A, Pilarska J, Sieradzka P, Szul M, Czuba B, et al. The impact of COVID-19 on female sexual health. Int J Environ Res Public Health. 2020;17(19):1.

54. Luetke M, Hensel D, Herbenick D, Rosenberg M. Romantic relationship conflict due to the COVID-19 pandemic and changes in intimate and sexual behaviors in a nationally representative sample of American adults. J Sex Marital Ther. 2020;46(8):747-62.

55. Micelli E, Cito G, Cocci A, Polloni G, Russo Gl, Minervini A, et al. Desire for parenthood at the time of COVID-19 pandemic: an insight into the Italian situation. J Psychosom Obstet Gynecol. 2020;41(3):183-90.

56. Gambir K, Garnsey C, Necastro KA, Ngo TD. Effectiveness, safety and acceptability of medical abortion at home versus in the clinic: a systematic review and meta-analysis in response to COVID-19. BMJ Glob Health. 2020;5(12):e003934

57. Moreau C, Shankar M, Glasier A, Cameron S, Gemzell-Danielsson K. Abortion regulation in Europe in the era of COVID-19: a spectrum of policy responses. BMJ Sex Reprod Health. 2020;47:e14.

58. McNicholas C, Swor E, Wan L, Peipert JF. Prolonged use of the etonogestrel implant and levonorgestrel intrauterine device: 2 years beyond Food and Drug Administration-approved duration. Am J Obstet Gynecol. 2017;216(6):586.

59. Smith KF, Goldberg M, Rosenthal S, Carlson L, Chen J, Chen C, et al. Global rise in human infectious disease outbreaks. J R Soc Interface. 2014;11(101):20140950.

60. Ghebreyesus TA, Kanem N. Defining sexual and reproductive health and rights for all. Lancet. 2018;391(10140):2583-5.

61. Patel J, Nielsen F, Badiani A, Assi S, Unadkat V, Patel B, et al. Poverty, inequality and COVID-19: the forgotten vulnerable. Public Health. 2020;183:110

62. Lokot M, Avakyan Y. Intersectionality as a lens to the COVID-19 pandemic: implications for sexual and reproductive health in development and humanitarian contexts. Sex Reprod Health Matters. 2020;28(1):1764748.

63. Ott MA, Bernard C. Preserving the reproductive rights of girls and women in the era of COVID-19: the need for a least restrictive solution. Am J Bioeth. 2020:20(7):120-2.

64. Laurencin CT, McClinton A. The COVID-19 pandemic: a call to action to identify and address racial and ethnic disparities. J Racial Ethnic Health Disparities. 2020;18:1-5.

65. Eliopoulos GM, Harris AD, Bradham DD, Baumgarten M, Zuckerman $I H$, Fink JC, et al. The use and interpretation of quasi-experimental studies in infectious diseases. Clin Infect Dis. 2004;38(11):1586-91.

66. Kim C, Fønhus M, Ganatra B. Self-administration of injectable contraceptives: a systematic review. BJOG. 2017;124(2):200-8.

67. Senderowicz L, Higgins J. Reproductive autonomy is nonnegotiable, even in the time of COVID-19. Int Perspect Sex Reprod Health. 2020;46:147-51.

\section{Publisher's Note}

Springer Nature remains neutral with regard to jurisdictional claims in published maps and institutional affiliations. 